\title{
Let-7b regulates alpaca hair growth by downregulating ectodysplasin A
}

\author{
NING LIU, SHU NIU, XIAO-RUI CAO, JIA-QI CHENG, SHU-YUAN GAO, \\ XIU-JU YU, HAI-DONG WANG, CHANG-SHENG DONG and XIAO-YAN HE \\ Alpaca Bioengineering Laboratory, College of Animal Science and Veterinary Medicine, \\ Shanxi Agricultural University, Taigu, Shanxi 030801, P.R. China
}

Received September 11, 2016; Accepted August 24, 2017

DOI: $10.3892 / \mathrm{mmr} .2018 .8442$

\begin{abstract}
Hypohidrotic ectodermal dysplasia (HED), also known as anhidrotic ectodermal dysplasia, is characterized by the clinical manifestations of less sweat or no sweat, sparse or no hair, tooth agenesis and/or abnormal tooth morphology. The characteristics of alpaca ear hair differ from the back hair. The ectodysplasin A (EDA) signaling pathway has a regulatory effect on skin development and hair growth. The aim of the present study was to study the effects of EDA on alpaca hair growth by examining the mRNA and protein expression levels of EDA in alpaca ear and back skin by reverse transcription-quantitative polymerase chain reaction and western blot analysis, respectively. Results indicated that EDA expression was higher in the ear skin compared with the back skin. The expression levels of let-7b in the skin of healthy alpacas varies; the difference between let-7b expression levels of the ear and back have been reported to be $>2$-fold, suggesting a role for let-7b in the development of adult alpaca skin and hair follicles. A dual-luciferase reporter vector was constructed to verify the targeting relationship between microRNA let-7b and EDA, and the results revealed that EDA was a target gene of let-7b. Alpaca skin fibroblasts were transfected with a let-7b eukaryotic expression vector to investigate the regulatory relationship between let-7b and EDA. The expression of EDA was decreased in the transfected group; immunocytochemical results demonstrated that the EDA protein was abundantly expressed in the fibroblast cytoplasm. EDA protein expression was weaker in the transfected cells than in the untransfected cells. These results suggested that EDA may serve a role in alpaca hair growth and is probably a target gene of let-7b; let-7b downregulated EDA mRNA and protein expressions,
\end{abstract}

Correspondence to: Professor Xiao-Yan He, Alpaca Bioengineering Laboratory, College of Animal Science and Veterinary Medicine, Shanxi Agricultural University, 1 Ming Xian South Road, Taigu, Shanxi 030801, P.R. China

E-mail: sxndhxy@163.com

Key words: hypohidrotic ectodermal dysplasia, anhidrotic ectodermal dysplasia, alpaca, microRNA, let-7b, hair growth which suggested that let-7b may regulate alpaca hair growth. These conclusions suggested that let-7b may be associated with HED.

\section{Introduction}

The hair follicles of mammals have been reported to be the only organ that continually cycles in growth, which includes the growth period (anagen), the return period (catagen) and the quiescence period (telogen) (1). Alpaca hair has a high economic value; therefore, a number of studies have investigated the mechanisms underlying the growth and color formation of alpaca hair (2-4). Previous studies have determined that microRNAs (miRNAs) serve important roles in the development and differentiation of skin and that miRNAs are associated with various skin diseases and skin cancers (5); however, miRNAs function in animal hair growth by regulating the expression of target genes. A previous study identified 39 significantly differentially expressed miRNAs in the ear and back skin of young alpaca, including let-7b. Through three target prediction software TargetScan (http://targetscan. org/), Pictar (http//pictar.bio.nyu.edu/) and miRbase targets (http:///microrna.sanger.ac uk/targets/v1/), Ectodysplasin A (EDA) was predicted to be a target gene of let-7b (6).

The EDA gene is located on chromosome Xq12-13 and belongs to the tumor necrosis factor superfamily, as revealed by the genetic analysis of EDA from syngeneic mice (7). EDA mutations may lead to a syndrome that causes ectodermal dysplasia without sweating, called hypohidrotic ectodermal dysplasia (HED). HED comprises $>180$ genetic syndromes and affects at least two ectodermal structures, including the hair, teeth, nails or exocrine glands (8). The most common type of ectodermal dysplasia is X-linked HED, which is characterized by sparse or absent hair, dental dysplasia or missing teeth, reduced sweating ability and defects of various lipid- or mucus-secreting glands. EDA is crucial for morphogenesis of skin appendages, including hair, teeth, sweat glands and eyelid glands (9). The EDA signaling pathway serves an important role in the formation of ectoderm, hair, teeth and exocrine glands (10). Enhanced EDA signals were reported to enlarge wool fibers and alter coat shape in mammals $(11,12)$. A previous study demonstrated that EDA mRNA expression 
was significantly higher in hair follicles in goat skin tissue during the catagen phase compared with expression during the telogen and anagen phases, and that expression was higher during the anagen phase compared with the telogen phase (13). This result indicated that EDA may affect the regulation of the hair follicle growth cycle.

Alpaca ear hair differs in characteristics from back hair: Ear hair is thick, straight and has a long growth cycle, whereas back hair is elongated, bent and has a short growth cycle (14). Gene chip analyses indicated that let-7b expression was significantly higher in the back skin compared with the ear skin of the alpaca; boinformatics analysis predicted EDA as a target gene of let-7b (6). In the present study, the differential expression of EDA in alpaca ear and back skin tissues was detected to analyze the effects of EDA on alpaca hair growth. In addition, a dual-luciferase reporter assay was used to verify that EDA was a target of let-7b, and the regulatory relationship between let-7b and EDA was verified at the cellular level. The present study provided additional information on the mechanisms underlying alpaca hair growth and may serve as a theoretical basis to continue exploring let-7b and its target genes. In addition, research into the regulation of let-7b on alpaca hair growth through downregulation of EDA may also inform further studies into HED.

\section{Materials and methods}

Animals, cells and plasmids. A total of 3 three-years-old male alpacas were obtained from an alpaca breeding base in Shanxi, China. Animal care and skin sample collection were in accordance with the International Guiding Principles for Biomedical Research Involving Animals (http://www.encyclopedia.com/science/encyclopedias-almanacstranscripts-and-maps/international-guiding-principles-biomedicalresearch-involving-animals) and approved by the Animal Experimentation Ethics Committee of Shanxi Agricultural University (Taigu, China). Two skin samples were collected from the ear and back ( $n=2 /$ site) of each alpaca: One biopsy sample was selected for RNA extraction, and the other biopsy sample was selected for protein extraction; all samples were soaked in liquid nitrogen.

Alpaca fibroblasts and 293T cells were stored in liquid nitrogen at the Alpaca Biological Laboratory in Shanxi Agricultural University (Taigu, China) and the primary alpaca fibroblasts were cultured in Alpaca Biological Laboratory, Shanxi Agricultural University. 293T cells were obtained from Shanghai Bioleaf Biotech Co., Ltd. (Shanghai, China). Alpaca fibroblasts and $293 \mathrm{~T}$ cells were cultured in $37^{\circ} \mathrm{C}, 5 \%$ $\mathrm{CO}_{2}$, in Alpaca fibroblasts medium (Thermo Fisher Scientific, Inc., Waltham, MA, USA) and Dulbecco's modified Eagle's medium (Thermo Fisher Scientific, Inc.), respectively.

The pmirGLO dual-luciferase miRNA target expression vector and the negative control (empty vector) were purchased from Promega Corporation (Madison, WI, USA). The pcDNA6.2-GW/EmGFP-let-7b overexpression vector based on the let-7b mature sequence; the negative control (empty vector) did not contain the let-7b sequence. The vectors were obtained from a previous deep sequencing analysis on alpaca skin (15) and were purchased from Invitrogen (Thermo Fisher Scientific, Inc., Waltham, MA, USA).
RNA extraction and reverse transcription-quantitative polymerase chain reaction $(R T-q P C R)$. Total RNA was isolated from the six skin samples (diameter $=8 \mathrm{~mm}$; three samples each from the ear skin and the back skin) and the transfected and untransfected alpaca fibroblast cells by using TRIzol LS Reagent (Invitrogen; Thermo Fisher Scientific, Inc.) according to the manufacturer's protocols. The specific transfection method is mentioned below in 'Alpaca fibroblast culture and transfection of the let-7b eukaryotic expression vector'. Total RNA concentration was determined with a NanoDrop 1000 spectrophotometer (NanoDrop Technologies; Thermo Fisher Scientific, Inc.), and the integrity of total RNA was confirmed by $1 \%$ agarose gel electrophoresis and ethidium bromide staining for $5 \mathrm{~min}$ at room temperature, then rinse with water at room temperature. cDNA was synthesized using a PrimeScript II First-Strand cDNA Synthesis kit (Takara Biotechnology Co., Ltd., Dalian, China) according to the manufacturer's protocols. The PCR product of EDA was 202 bp, as confirmed by $1 \%$ agarose gel and 2000 DNA Marker; the EDA gene was sequenced by the Beijing Genomics Institute (Shenzhen, China). Gene sequencing analysis revealed that the alpaca EDA gene was homologous with other species [published on National Center for Biotechnology Information (NCBI), Bethesda, MD, USA] by NCBI-BLAST https://blast .ncbi.nlm.nih.gov/Blast.cgi; accession nos. XM_015250693.1 XM_014566996.1, XM_010978765.1, XM_010973676.1, XM_008155957.1, XM_008155955.1, XM_004871764.2, XM_004871761.2,XM_012643114.1 and XM_012643097.1. Then qPCR was conducted with SYBR Premix Ex Taq II (Perfect Real Time; Takara Biotechnology Co., Ltd.) according to the manufacturer's protocols. The primer sequences used were: EDA, forward, 5'-GTGGACGGCACCTACTTCATC TAT-3', reverse: 5'-GCACCATCTTCACAGCGATCTTCT-3'; EDA expression was normalized to $18 \mathrm{~S}$ rRNA. The reaction conditions constituted pre-denaturation: $95^{\circ} \mathrm{C}$ for $10 \mathrm{~min}$; denaturation: $95^{\circ} \mathrm{C}$ for $15 \mathrm{sec}$; annealing: $63^{\circ} \mathrm{C}$ for $30 \mathrm{sec}$; extension $72^{\circ} \mathrm{C} 30 \mathrm{sec} ; 40$ cycles; $95^{\circ} \mathrm{C} 15 \mathrm{sec}, 60^{\circ} \mathrm{C} 1 \mathrm{~min}$ and $95^{\circ} \mathrm{C}, 15 \mathrm{sec}$. and the relative expression level was calculated using the $2^{-\Delta \Delta \mathrm{Cq}}$ method (16).

Western blot analysis. Total protein content from the six skin samples (diameter $=8 \mathrm{~mm}$; three samples each from the ear skin and the back skin), the transfected and untransfected alpaca fibroblasts were extracted with a protein extraction kit (radioimmunoprecipitation lysis buffer, Beyotime Institute of Biotechnology, Haimen, China) according to the manufacturer's protocols. The specific transfection method is mentioned below in 'Alpaca fibroblast culture and transfection of the let-7b eukaryotic expression vector'. The protein concentrations were determined using a NanoDrop 1000 spectrophotometer (NanoDrop Technologies; Thermo Fisher Scientific, Inc.). Equal amounts (300 $\mu \mathrm{g} /$ lane) of protein from alpaca skin samples and equal amounts (500 $\mu \mathrm{g} / \mathrm{lane})$ of protein from cells were separated by $10 \%$ SDS-PAGE and transferred onto polyvinylidene fluoride membranes (constant pressure $100 \mathrm{~V}, 1 \mathrm{~h}$ ). The membranes were blocked with 5\% nonfat milk (w/v) in TBST (TBS with $0.1 \%$ Tween 20) at room temperature for $1 \mathrm{~h}$. Membranes were incubated with primary antibodies against EDA (1:200, ab125233, Abcam, Cambridge, UK) and anti $\beta$-actin antibody (1:1,500; CW0096 M, CWBIO, 
Beijing, China) at $4^{\circ} \mathrm{C}$ overnight. Following washing in TBST, membranes were incubated with the goat anti-rabbit horseradish-peroxidase (HRP) secondary antibody (1:8,000 in 3\% TBST; bs-0295G-HRP, BIOSS, Beijing, China) and the goat anti-mouse (HRP) secondary antibody (1:5,000 in 3\% TBST; bs-0296G-HRP, BIOSS) for $1 \mathrm{~h}$ at $37^{\circ} \mathrm{C}$. Membranes were washed with TBST and protein bands were visualized with an Enhanced Chemiluminescence Western Blot kit (CWBIO). EDA signal intensity was quantified using ImageJ (Image J Launcher 1.4.3.67, National Institutes of Health, Bethesda, MD, USA) and Quantity One software (Quantity One 4.6.2.70, Bio-Rad Laboratories Inc, Hercules, CA, USA); relative expression levels were normalized to $\beta$-actin.

Dual-luciferase reporter assay. The 3'-untranslated region (UTR) of EDA from Alpaca back and ear skin samples was amplified by PCR using the following primers to construct the dual-luciferase vector pmirGLO-EDA-3'-UTR: Forward (SacI restriction site underlined) 5'-CGAGCTCGACCC TGAATCCGTACTTGG-3', reverse (XbaI restriction site underlined) 5'-GCTCTAGAGCAAATCCCTCTGTCCTAT CCTC-3'. The reaction conditions were: Pre-denaturation: $94^{\circ} \mathrm{C}, 2 \mathrm{~min}$; denaturation: $94^{\circ} \mathrm{C}, 30 \mathrm{sec}$; annealing $53^{\circ} \mathrm{C}$, $30 \mathrm{sec}$; extension $72^{\circ} \mathrm{C}, 30 \mathrm{sec} ; 35$ cycles; end extension: $72^{\circ} \mathrm{C}, 2 \mathrm{~min}$. A $2 x \mathrm{Taq}$ MasterMix (CWBIO) was used, and composed of Es Taq DNA Polymerase, $\mathrm{Mg} 2+$, dNTPs and PCR stabilizer and enhancer. The amplified product (469 bp) was connected to the T-Vector pMD19 (Takara Biotechnology Co., Ltd.), and then the EDA-3'-UTR-T-Vector pMD19 and the pmirGLO vector (Promega Corporation) were double digested by the $S a c \mathrm{I}$ and $\mathrm{XbaI}$. Finally, the two digested products were ligated with T4 ligase. The dual-luciferase recombinant vector was constructed. The empty pmirGLO vector $(368.7 \mu \mathrm{g} / \mu \mathrm{l})$ and let- $7 \mathrm{~b}$ negative control (349.6 ng/ul) served as negative controls. Untransduced $293 \mathrm{~T}$ cells were plated in 24-well plates to $\sim 60 \%$ confluence and transfected with the pmirGLO-EDA-3'-UTR (422.3 ng/ $\mu \mathrm{l}$; pmirGLO-EDA) vector and the pcDNA6.2-GW/EmGFP-let-7b (348.4 ng/ $\mu \mathrm{l})$ overexpression vector using the Attractene Transfection Reagent (Qiagen, Shanghai, China). Dual-luciferase assays (Promega Corporation) were performed $48 \mathrm{~h}$ post-transfection according to the manufacturer's protocols, and luciferase activity was determined using a GloMax96 Microplate Luminometer (Promega Corporation). Firefly and Renilla luciferase served as the experimental and control, respectively. The firefly/Renilla ratios were normalized to a negative control and that of an empty plasmid control.

Alpaca fibroblast culture and transfection of the let-7b eukaryotic expression vector. Alpaca fibroblasts were cultured in six-well plates at $37^{\circ} \mathrm{C}$ and $5 \% \mathrm{CO}_{2}$ to $\sim 60 \%$ confluence; the cells were transfected with $1.2 \mu \mathrm{g}$ of the let-7b expression vector (treatment group) by using the Attractene Transfection Reagent (Qiagen) according to the manufacturer's protocols. Untransfected cells (cell control) incubated in Attractene Transfection Reagent (Qiagen) and cells transfected with let-7b negative control expression vectors served as negative controls. Following $48 \mathrm{~h}$ incubation, fluorescence from the GFP was observed under a fluorescence microscope (Leica Microsystems $\mathrm{GmbH}$ ). The cells were collected for total RNA

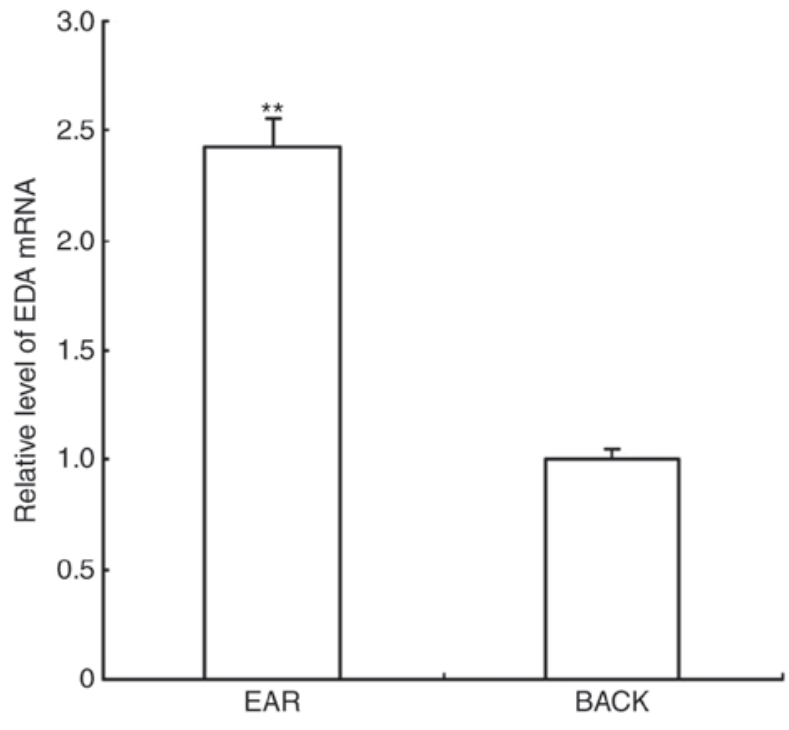

Figure 1. Analysis of relative EDA mRNA expression in alpaca ear and back skin. $2^{-\triangle \Delta C q}$ data analysis of EDA abundance was normalized to $18 \mathrm{~S}$ rRNA abundance. The relative expression of EDA mRNA in alpaca ear skin was 2.425 times higher than expression in back skin. Data are presented as the mean \pm standard deviation; ${ }^{* *} \mathrm{P}<0.01$. EDA, ectodysplasin A.

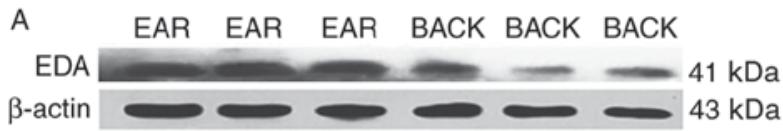

B

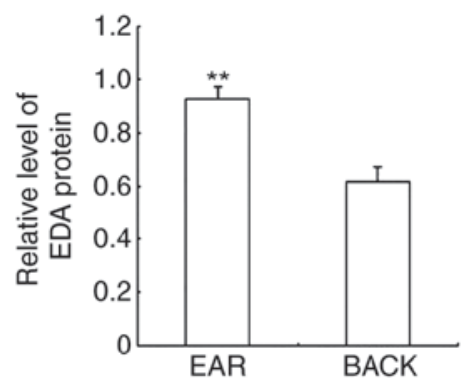

Figure 2. Western blot analysis of the relative protein expression of EDA in alpaca ear and back skin. (A) Representative western blot analysis demonstrating the different band intensities of EDA protein expression from the two tissues. (B) Data analysis of EDA levels normalized to $\beta$-actin levels; data are presented as the mean \pm standard deviation; ${ }^{* *} \mathrm{P}<0.01$. EDA, ectodysplasin $\mathrm{A}$.

and protein extraction for the analysis of EDA expression by RT-qPCR and western blotting, respectively as aforementioned.

Immunocytochemical analysis of EDA in fibroblasts. Alpaca fibroblasts were seeded onto slides in 24 -well plates at $37^{\circ} \mathrm{C}$ and $5 \% \mathrm{CO}_{2}$ to $\sim 60 \%$ confluence. The cells were transfected with $0.4 \mu \mathrm{g}$ of the let-7b expression vector (treatment group) by using the Attractene Transfection Reagent (Qiagen) according to the manufacturer's protocols, and cells incubated in Attractene Transfection Reagent (Qiagen) served as an untransfected control. Following $48 \mathrm{~h}$ incubation $\left(37^{\circ} \mathrm{C}\right.$, $5 \% \mathrm{CO}_{2}$ ), the fibroblasts were processed for immunocytochemistry as previously described (17). Cells were fixed with $4 \%$ paraformaldehyde for $30 \mathrm{~min}$ at room temperature, incubated in $3 \%$ hydrogen peroxide at $37^{\circ} \mathrm{C}$ for $10 \mathrm{~min}$, 


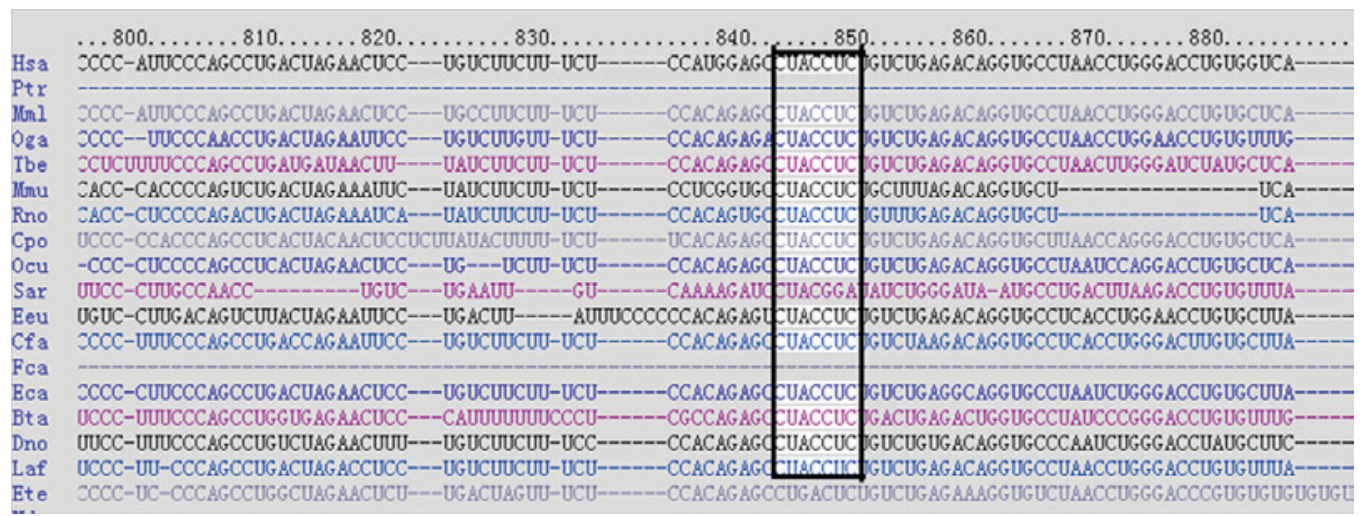

Figure 3. Schematic diagram of let-7b target binding site in 3'-UTR of EDA.

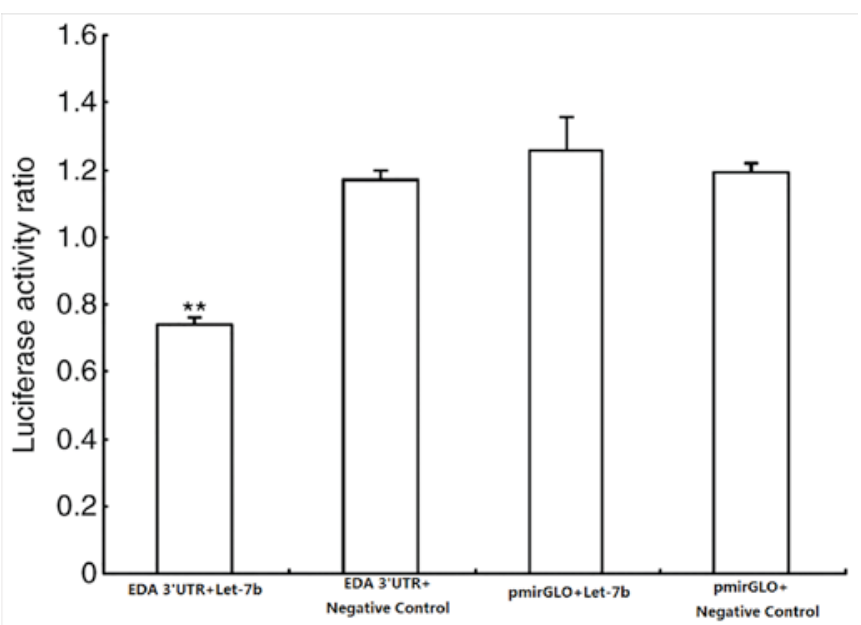

Figure 4. Relative luciferase activity following co-transfection of let-7b over-expression vector and dual-luciferase reporter plasmid for EDA 3'-UTR. The ratio of the experimental group decreased compared with that of the control groups. Data are presented as the mean \pm standard deviation; ${ }^{* *} \mathrm{P}<0.01$. EDA, ectodysplasin A; UTR, untranslated region.

blocked with $5 \%$ bovine serum albumin (Beijing Solarbio Science \& Technology, Beijing, China) at $37^{\circ} \mathrm{C}$ for $30 \mathrm{~min}$ and incubated with anti-EDA rabbit polyclonal antibody (1:30, ab198022, Abcam Cambridge, UK) at $4^{\circ} \mathrm{C}$ for $14 \mathrm{~h}$; cells only for the negative staining control were incubated in PBS in similar conditions. HRP-conjugated goat anti-rabbit immunoglobulin G (SP Rabbit HRP kit, CWBIO) was used as a secondary antibody and cells were incubated at $37^{\circ} \mathrm{C}$ for 30 min. 3-3'diaminobenzidine (DAB) was used with the SP Rabbit HRP kit (CWBIO), which served as a positive control, containing DAB-A (20x) and DAB-B (1x) reagents; DAB produces a brown precipitate following catalysis by HRP. Cells of the -positive control group were incubated in DAB $\sim 3$ min in the dark at room temperature and subsequently counterstained with hematoxylin for $\sim 5 \mathrm{~min}$ at room temperature. The slides were mounted, cover-slipped and evaluated under a Leitz DMRB microscope (Leica Microsystems $\mathrm{GmbH}$ ), five fields were examined per slide.

Statistical analysis. Data are presented as the mean \pm standard deviation of three replicates. Data were analyzed using SPSS v17.0 software (SPSS, Inc., Chicago, IL, USA).

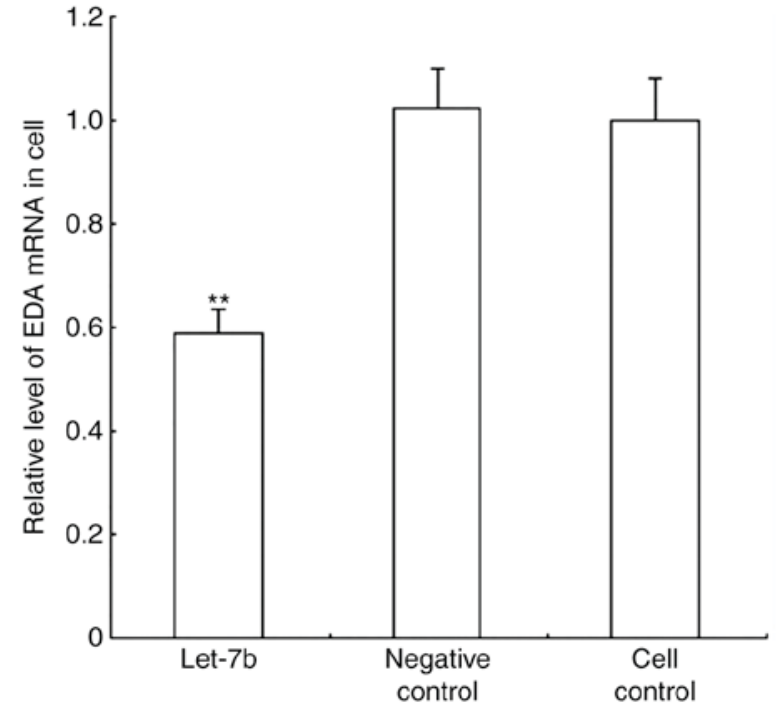

Figure 5. Alpaca fibroblasts were transfected with the let-7b overexpression vector and let-7b negative control, and EDA mRNA expression was examined in the transfected and untransfected cells (cell control) by reverse transcription-quantitative polymerase chain reaction. EDA mRNA expression levels were normalized to $18 \mathrm{~S}$ rRNA. The results indicated that let- $7 \mathrm{~b}$ overexpression significantly decreased EDA mRNA expression. Data are presented as the mean \pm standard deviation; ${ }^{* *} \mathrm{P}<0.01$. EDA, ectodysplasin $\mathrm{A}$.
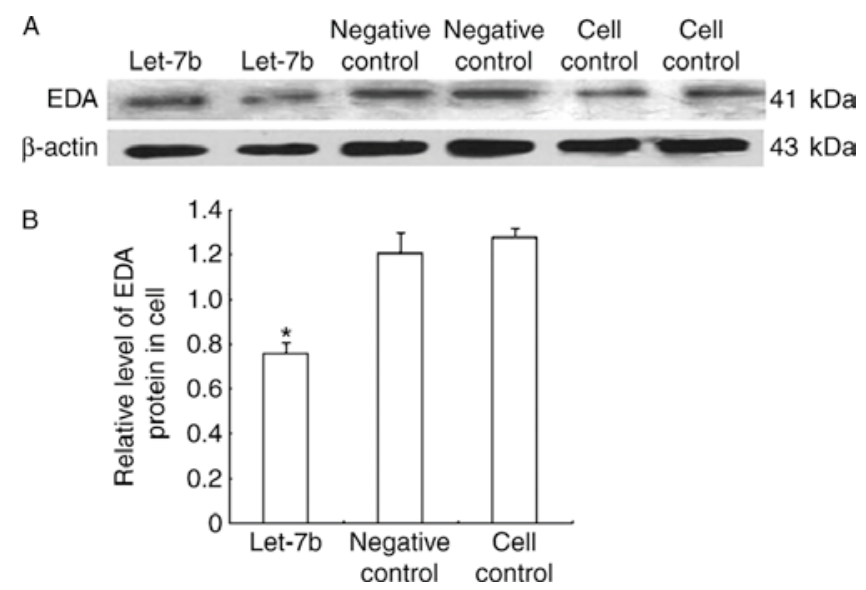

Figure 6. Western blot analysis of the relative protein expression levels of EDA in different groups. (A) Representative western blot analysis demonstrating the different band intensities of EDA protein expression from the two tissues. (B) Data analysis of EDA levels normalized to $\beta$-actin; data are presented as the mean \pm standard deviation; ${ }^{*} \mathrm{P}<0.05$. EDA, ectodysplasin A. 


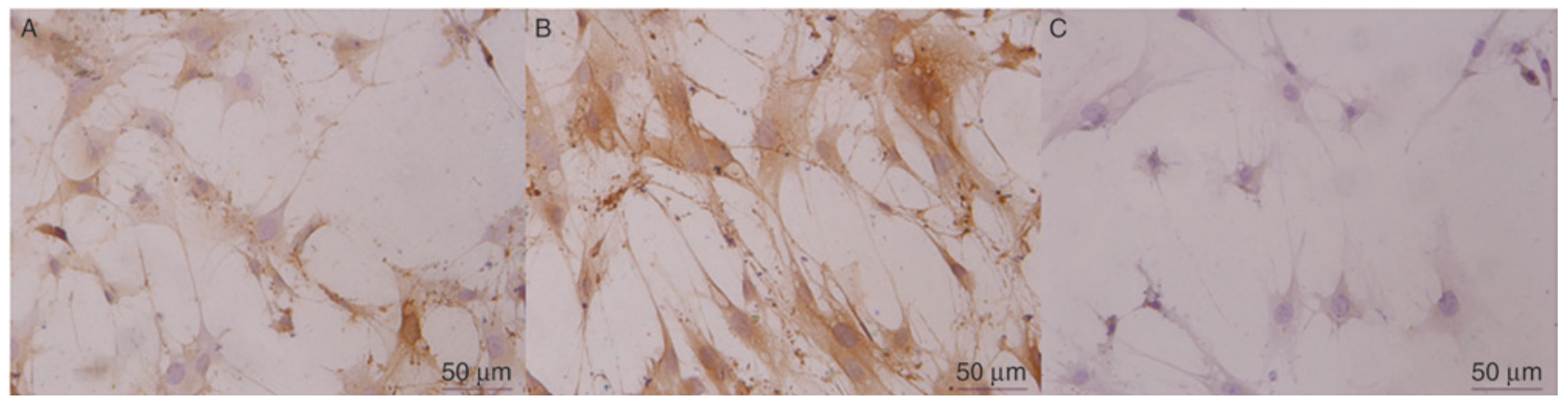

Figure 7. Immunocytochemical staining results of EDA expression. (A) Cell was transfected with let-7b positive; magnification, x20. (B) Untransfected cell control; magnification, x20. (C) Negative staining control cell; magnification, x20. Positive reactivity was observed predominantly as fibroblast cytoplasmic staining. Fibroblasts in the negative staining control group exhibited no specific immunostaining. The positive reaction of the EDA protein was weaker in the transfected cells than in the untransfected cells. EDA, ectodysplasin A.

Multiple comparison analyses were performed by one-way analysis of variance followed by a Newman-Keuls post hoc test. $\mathrm{P}<0.05$ was considered to indicate a statistically significant difference.

\section{Results}

RT-qPCR analysis of EDA in alpaca ear and back skin. RT-qPCR results demonstrated that the amplification curves of EDA and 18S rRNA were smooth; the inflection points were clear with marked repeatability, and the melting curves revealed a single peak, indicating that there was no nonspecific amplification during the amplification process. EDA mRNA expression levels in ear skin were relatively 2.425 higher compared with expression in the back skin (Fig. 1).

Western blot analysis of EDA in alpaca ear and back skin. EDA protein expression levels were detected in alpaca ear and back skin by western blot. The molecular size of the EDA protein was $41 \mathrm{kDa}$, whereas that of the $\beta$-actin protein was $43 \mathrm{kDa}$ (Fig. 2A). The relative protein expression levels in the ear and back skin were $0.9254 \pm 0.0473$ and $0.6145 \pm 0.0582$, respectively. EDA protein expression in alpaca ear skin was 1.506 -fold higher compared with expression in back skin (Fig. 2B).

Dual-luciferase reporter assay. Our previous study reported that let-7b may be involved in regulating hair growth and was differentially expressed in alpaca skin from various parts of the body with different fiber qualities (6). Previous bioinformatics predictions using the publicly available algorithm from TargetScan release 4.1 (www.targetscan.org) indicated that EDA may be a target of let-7b (Fig. 3) (6). In the present study, a dual-luciferase recombinant vector that contained the 3'-UTR of EDA was constructed by combining target prediction algorithms. The firefly/Renilla ratio collected from the experimental group (cells transfected with pmirGLO-EDA-3'-UTR and let-7b overexpression vector) significantly decreased, whereas of the control groups (cells transfected with pmirGLO and let-7b negative controls) did not change (Fig. 4).

EDA expression in alpaca fibroblasts overexpressing let-7b. Alpaca fibroblasts were transfected with the let-7b overexpression and negative control vectors, and cultured under standard conditions, to further evaluate let-7b regulation of EDA. Relative EDA mRNA expression levels were determined by RT-qPCR (Fig. 5), and protein expression levels were detected through western blot analysis (Fig. 6). The results demonstrated that let-7b overexpression resulted in decreased EDA expression at both the mRNA and protein level. let-7b transfected cells exhibited a 1.914-fold reduction in EDA mRNA quantity compared with the control groups, whereas the EDA protein expression level was 1.589 -fold lower in the let-7 overexpressing cells, compared with the control groups.

EDA expression in fibroblast cells. Alpaca fibroblasts were transfected with the let-7b overexpression vector. Positive reactivity was noted as predominantly fibroblastic cytoplasmic staining, and the negative staining control cells exhibited no specific immunostaining of fibroblasts as EDA was not expressed (Fig. 7). Immunoreactivity staining was barely detected in the let-7b overexpression vector-transfected group, which was consistent with the results of RT-qPCR and western blotting.

\section{Discussion}

The first miRNA was discovered in a nematode in 1993, and numerous miRNAs have subsequently been identified in a variety of species (18-27); an increasing number of reports on miRNA represent a new approach of gene regulation. miRNAs regulate the expression of target genes in two ways, degradation and inhibition of expression. miRNA (miR)-17, let-7, miR-30 and miR-8 are expressed in mouse, goat and sheep skin; these miRNAs may be related to skin development (28). Fibroblast growth factor (FGF) 5 and transforming growth factor $\beta$ receptor type I (TGF $\beta$ RI) have been previously identified as target genes of let-7b. Let-7b negatively regulates its target genes by binding to the $3^{\prime}$ end of the mRNA $(29,30)$. FGF5 serves an important role in the transformation process during the early-mid growth period of hair follicles. FGF5 overexpression inhibits the growth of hair length. In the hair follicle cycle period, TGF $\beta R I$ is highly expressed in the hair follicle root sheath, which aids in starting and maintaining the hair follicle catagen $(29,30)$. EDA was predicted as a target gene of let-7b (6).

Previous genetic studies demonstrated that the EDA/EDA receptor signaling pathway serves an important role in the development of skin appendages (31). Regulation of the EDA 
signaling pathway is complex, and previous studies have reported that sonic hedgehog, Wnt/Dickkopf, bone morphogenetic protein and lymphotoxin $\beta$ are located downstream of EDA signaling $(32,33)$. Some of these downstream genes were revealed to inhibit the development of skin appendages, whereas other genes only inhibited the development of specific organs, and yet others are only expressed at a given period; these data indicated that EDA had a wide range of time and organ specificity during skin development (34). For example, EDA signaling may regulate the hair follicle transition from the growth phase to the catagen phase; in addition, EDA signaling serves a role in hair follicle morphogenesis and regulates the apoptosis of hair follicle keratinocytes in the catagen phase. EDA knockout or the pharmacological inhibition of EDA signalling within wild type mice may accelerate the growth of the hair follicle catagen (35). Alpaca ear hair is thick and straight, and has a long growth cycle, whereas back hair is elongated, bent and has a short growth cycle (14). To study the role of EDA in the growth of alpaca hair, differential expression of EDA in alpaca ear and back skin was examined, and the results demonstrated that EDA expression in alpaca ear skin was significantly higher compared with expression in back skin. These results suggested that the growth cycle of the ear and back hair of alpaca may be associated with the differential expression of EDA in the ear and back skin of alpaca.

Results from the present study verified that EDA expression was higher in the ear compared with expression in the back skin, however the expression of let-7b was reported to be significantly higher in the back compared with expression in the ear skin and EDA was predicted to be a target gene of let-7b (6). Let-7b probably regulates EDA expression by binding with the EDA 3'-UTR. The present study successfully amplified the EDA 3'-UTR and used this sequence to construct the EDA dual-luciferase reporter vector, the results of which confirmed that EDA was the target gene of let-7b. Following transfection of the let-7b eukaryotic overexpression vector into alpaca skin fibroblasts, the mRNA and protein expression levels of EDA decreased. Immunocytochemical analysis of EDA expression in the let-7b-transfected and untransfected groups demonstrated that the EDA protein was abundantly expressed in the fibroblast cytoplasm and that expression in the transfected cells was weaker compared with expression in the untransfected cells. These results demonstrated that let-7b negatively regulated EDA at the mRNA and protein levels.

miRNAs serve important roles in the incidence of disease, as well as cell differentiation, cell growth, apoptosis and immune response (36-38). EDA is a target gene of let-7b, and EDA mutations may lead to HED (8), which indicated that let-7b may be associated with HED; however, further research is required. In conclusion, EDA was demonstrated to be differentially expressed in alpaca ear and back skin, which suggested that EDA may serve role in the growth cycle of alpaca hair. In addition, EDA is the target gene of let-7b, and let-7b negatively regulates EDA mRNA and protein.

\section{Acknowledgements}

The present study was supported by The National Natural Science Foundation of China (grant nos. 31172283 and 31302049).

\section{References}

1. Wang N, Rong EG and Yan XH: Research progress of hair follicle development and hair production. J Northeast Agric Univ 43: 6-11, 2012.

2. Zhu Z, He J, Dong C, Jiang J, Bai R, Yu X, Lv L, Fan R, He X, Geng J, et al: MicroRNA-25 functions in regulation of pigmentation by targeting the transcription factor MITF in alpaca (Lama pacos) skin melanocytes. Domest Anim Endocrinol 38: 200-209, 2010.

3. Dong Y, Cao J, Wang H, Zhang J, Zhu Z, Bai R, Hao H, He X, Fan $R$ and Dong C: Nitric oxide enhances the sensitivity of alpaca melanocytes to respond to alpha-melanocyte-stimulating hormone by up-regulation melanocortin-1 receptor. Biochem Biophys Res Commun 396: 849-853, 2010.

4. Geng JJ, Mu XL, Suan LT, Jiang JB, Zhang J, Li HQ, Zhang Y and Dong CS: Expression and immunolocalization of endothelin receptor B in Alpaca skin of different colors. Acta Veterinaria et Zootechnica Sinica 41: 1478-1484, 2010.

5. Sand M, Gambichler T, Sand D, Skrygan M, Altmeyer P and Bechara FG: MicroRNAs and the skin: Tiny players in the body's largest organ. J Dermatol Sci 53: 169-175, 2009.

6. He XY, Hao HQ, Liu DD, Fan RW, Cao J, Zhu ZW, Dong YJ and Xing HQ: Difference of microRNA expression in the ear and back skin of Young Alpaca (Lama pacos). Chin J Biochem Mol Biol 26: 1016-1022, 2010.

7. Kere J, Srivastava AK, Montonen O, Zonana J, Thomas N, Ferguson B, Munoz F, Morgan D, Clarke A, Baybayan P, et al: $\mathrm{X}$-linked anhidrotic (hypohidrotic) ectodermal dysplasia is caused by mutation in a novel transmembrane protein. Nat Genet 13: 409-416, 1996.

8. Visinoni AF, Lisboa-Costa T, Pagnan NA and Chautard-Freire-Maia EA: Ectodermal dysplasias: Clinical and molecular review. Am J Med Genet A 149A: 1980-2002, 2009.

9. Pinheiro M and Freire-Maia N: Ectodermal dysplasias: A clinical classification and a causal review. Am J Med Genet 53: 153-162, 1994.

10. Clarke A, Phillips DI, Brown R and Harper PS: Clinical aspects of X-linked hypohidrotic ectodermal dysplasia. Arch Dis Child 62: 989-996, 1987.

11. Mustonen T, Pispa J, Mikkola ML, Pummila M, Kangas AT, Pakkasjärvi L, Jaatinen R and Thesleff I: Stimulation of ectodermal organ development by ectodyplasin-A1. Dev Biol 259: 123-136, 2003.

12. Zhang M, Brancaccio A, Weiner L, Missero C and Brissette J: Ectodysplasin regulates pattern formation in the mammalian hair coat. Genesis 37: 30-37, 2003.

13. Jiang W, Xue P, He YX and Chen YL: Molecular cloning, sequence analysis and expression of goat Eda gene. J Northwest A F Univ (Nat. Sci. Ed) 40: 7-12, 2012.

14. Liu DD, Zhang ZY, He XY, Dong YJ, Zhu ZW, Bai R, Zhang J, Hao HQ and Xing HQ: FGF5 expression and immunolocalization in back and ear skin of young alpaca (Lama pacos). Chin J Bioch Mol Biol 5: 473-479, 2011.

15. Tian X, Jiang J, Fan R, Wang H, Meng X, He X, He J, Li H, Geng J, Yu X, et al: Identification and characterization of microRNAs in white and brown alpaca skin. BMC Genomics 13: 555, 2012.

16. Livak KJ and Schmittgen TD: Analysis of relative gene expression data using real-time quantitative PCR and the 2(-Delta Delta C(T)) method. Methods 25: 402-408, 2001.

17. Cekanova M, Uddin MJ, Bartges JW, Callens A, Legendre AM, Rathore K, Wright L, Carter A and Marnett LJ: Molecular imaging of cyclooxygenase-2 in canine transitional cell carcinoma as in vitro and in vivo. Cancer Prev Res (Phila) 6: 466-476, 2013.

18. Lee RC, Feinbaum RL and Ambros V: The C. Elegans heterochronic gene lin-4 encodes small RNAs with antisense complementarity to lin-14. Cell 75: 843-854, 1993.

19. Lagos-Quintata M, Rauhut R, Lendeckel W and Tuschl T: Identification of novel genes coding for small expressed RNAs. Science 294: 853-858, 2001.

20. Lee RC and Ambros V: An extensive class of small RNAs in Caenorhabditis elegans. Science 294: 862-864, 2001.

21. Dostie J, Mourelatos Z, Yang M, Sharma A and Dreyfuss G: Numerous microRNPs in neuronal cells containing novel microRNAs. RNA 9: 180-186, 2003.

22. Lim LP, Glasner ME, Yekta S, Burge CB and Bartel DP: Vertebrate microRNA genes. Science 299: 1540, 2003. 
23. Lim LP, Lau NC, Weinsstein EG, Abdelhakim A Yekta S, Rhoades MW, Burge CB and Bartel DP: The microRNAs of Caenorhabditis elegans. Genes Dev 17: 991-1008, 2003.

24. Grad Y, Aach J, Hayes GD, Reinhart BJ, Church GM, Ruvkun G and Kim J: Computational and experimental identification of C. Elegans microRNAs. Mol Cell 11: 1253-1263, 2003.

25. Reinhart BJ, Weinsstein EG, Rhoades MW, Bartel B and Bartel DP: MicroRNAs in plants. Genes Dev 16: 1616-1626, 2002.

26. Lau NC,Lim LP, Weinsstein EG and Bartel DP: An abundant class of tiny RNAs with probable regulatory roles in Caenorhabditis elegans. Science 294: 858-862, 2001.

27. Kim J, Krichevsky A, Grad Y, Hayes GD, Kosik KS, Church G and Ruvkun G: Identification of many microRNAs that copurify with polyribosomes in mammalian neurons. Pro Natl Acad Sci USA 101: 360-365, 2004.

28. Liu DD, He XY and Hao HQ: The regulatory role of microRNAs in the development of animal skin and hair. Chin J Biochem Mol Biol 26: 802-808, 2010.

29. Wang T, Zhang Y, Wang HD, Shen Y, Liu N, Cao J, Yu XJ, Dong CS and He XY: Alpaca fiber growth is mediated by microRNA let-7b via down-regulation of target gene FGF5. Genet Mol Res 14: 13754-13763, 2015.

30. Yan S, Yu Z, Ning L, Hai-Dong W, Jian-Shan X, Shu-Yuan G, Jia-Qi C, Xiu-Ju Y, Ting W, Chang-Sheng D and Xiao-Yan H: Let-7b promotes alpaca hair growth via transcriptional repression of TGFßR I. Gene 577: 32-36, 2016.

31. Weih F and Caamaño J: Regulation of secondary lymphoid organ development by the nuclear factor-kappaB signal transduction pathway. Immunol Rev 195: 91-105, 2003.
32. Headon DJ, Emmal SA, Ferguson BM, Tucker AS, Justice MJ, Sharpe PT,Zonana J and Overbeek PA: Gene defect in ectodermal dysplasia implicates a death domain adapter in development. Nature 414: 913-916, 2001.

33. Cui CY and Schlessinger D: EDA signaling and skin appendage development. Cell Cycle 5: 2477-2483, 2006.

34. Botchkarev VA and Fessing MY: Edar signaling in the control of hair follicle development. J Investig Dermatol Symp Proc 10: 247-251, 2005

35. Alvarez-Garcia I and Miska EA: MicroRNA functions in animal development and human disease. Development 132: 4653-4662, 2005.

36. Naguibneva I, Ameyar-Zazoua M, Polesskaya A, Ait-Si-Ali S, Groisman R, Souidi M, Cuvellier S and Harel-Bellan A: The microRNA miR-181 targets the homeobox protein Hox-A11 during mammalian myoblast differentiation. Nat Cell Biol 8: 278-284, 2006

37. Jakymiw A, Lian SL, Eystathioy T, Li S, Satoh M, Hamel JC, Fritzler MJ and Chan KL: Disruption of GW bodies impairs mammalian RNA interference. Nat Cell Biol 7: 1267-1274, 2005.

38. Naguibneva I, Ameyar-Zazoua M, Nonne N, Polesskaya A Ait-Si-Ali S, Groisman R, Souidi M, Pritchard LL and Harel-Bellan A: An LNA-based loss-of-function assay for micro-RNAs. Biomed Pharmacother 60: 633-638, 2006. 